\title{
Integrated production management for production planning, loading and scheduling
}

Fuminori Oba, Takeshi Murayama and Dianying Ma

Faculty of Engineering, Hiroshima University

Kagamiyama 1-4-1, Higashi-Hiroshima, Hiroshima, 739, Japan

Tel: +81-824-24-7550 Fax: +81-824-22-7193

oba@mec.hiroshima-u.ac.jp

\begin{abstract}
This paper deals with a production planning system which is integrated with loading and scheduling in the job-shop type production circumstances. The production planning system is based on an MRP explosion system in which the leadtime of each part is given based on the processing time of the part in each work-center and the time-bucket size is adjusted by using the feedback information of the production load out of the loading system. In the loading system, the loading and the balancing activities are carried out for each work-center, and the time bucket is given by subdividing the one in production planning and adjusted by the result of scheduling. A numerical example is presented to illustrate the procedure of the proposed system.
\end{abstract}

\section{Keywords}

Production planning, loading, scheduling, integrated production control

\section{INTRODUCTION}

In the low volume, high variety production and fluctuating production environment, decision-making in the production management is complicated and 
much affects to production leadtime and costs. In such environment, the original MRP(Material Requirement Planning) system and even the MRP II(Manufacturing Resource Planning) system cause the following problems:

- Any explicit procedure is not presented to balance the workload and capacity at the loading and shop-floor control level.

- It is difficult to integrate production planning with loading and scheduling, because the MRP systems manage these planning problems with an identical bucket's span of time, though the appropriate time spans differ with the different levels in decision-making.

- Using a pre-determined leadtime for each part in the time-phased explosion process of the MRP often results in the overdue production or too much production period of time, because the leadtimes for all the parts vary with the production workload and the facility capacity available in the shop-floor and are to be first available after scheduling.

To cope with these problems, non-standard approaches to MRP II in which scheduling to finite capacity is included are presented in recent years( Scott, 1994). Matoba et al.(1993) proposed a dynamic MRP in which production planning is integrated with scheduling for a flow-shop type production system. These approaches contain the large feedback loops in which the results of scheduling are utilized for production planning. It is however very hard to apply these approaches to the job-shop type or cell-type production systems, because decision-making with such feedback loops is too complicated in the low volume, high variety production environment.

In this study, a new procedure is proposed to integrate production planning with loading and scheduling, which has basically the same architecture as the nonstandard MRP II systems, but is not so tightly bound by the feedback loops. This approach may lead not only to simple decision-making in the integrated production management, but also to the shorter leadtimes for whole production as well as to the elimination of the overdue productions. The production environment treated here is assumed that the low volume and many kinds of products are simultaneously produced through several job-shop type work-centers for machining and assembly, each of which comprises several machines.

\section{PRODUCTION PLANNING MODULE}

An architecture of the integrated production management proposed here is as shown in Figure 1, where the bold lines indicate the main routes of information for decision-making and the fine lines are feedback information which is given only if necessary.

Primary objectives of production planning are to determine the production periods of time and quantities for all products and component parts, according to the time-phased parts explosion technique in MRP using the bills of materials, and 
balancing the production workload in each work-center with the production capacity available in every period. The proposed system is therefore the decisionmaking tool, whereas the typical MRP II is essentially the decision support tool which gives the planner information on workload and capacity, and no information of how to balance them.

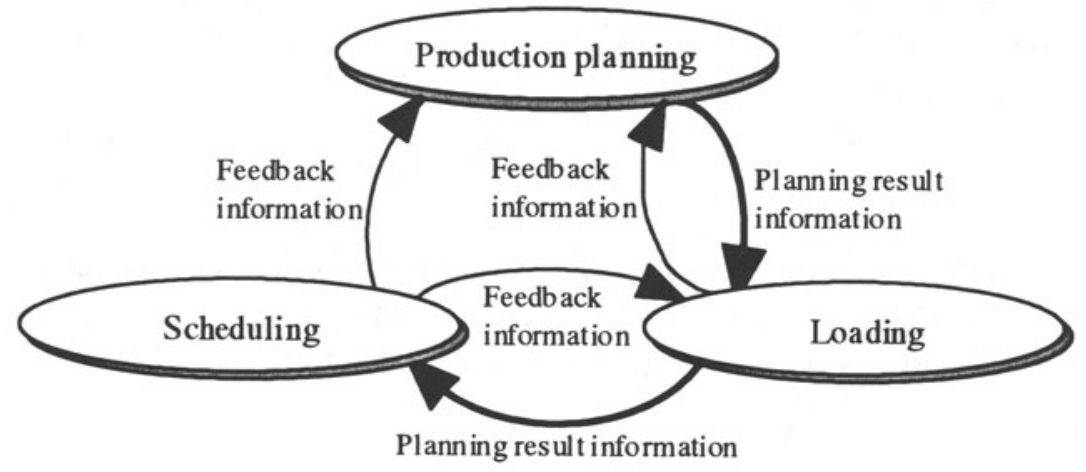

Figure 1 Architecture of integrated production management.

\subsection{MRP parts explosion process}

The span of time bucket adopted as the unit of time in MRP is determined by the planner taking account of the manufacturing environment and the firmness of demand over the future horizon, for example, a month, a week or a day. In the MRP II standard systems, once a time span is selected, the span is identically used in the loading and scheduling levels as well as the production planning level, in spite of that the appropriate spans may be different with the levels in the hierarchical structure of decision-making. This may cause the problem to make it difficult to integrate production planning with loading and scheduling. To cope with this problem, we appropriately adopt the different span of time bucket for loading and scheduling from production planning.

In the production planning level, the larger bucket-size associated with the large number of levels in the product structure trees results in the longer leadtime. On the other hand, the smaller bucket-size leads to frequent rescheduling to ensure the accuracy of the production schedule. In this study, a week is initially adopted as the span of time bucket in the production planning level, which may be adjusted later by using the feedback information of the production workload out of the loading system if necessary.

Next we consider the planning leadtime to be used for the time-phased parts explosion in MRP. In MRP standard systems, the planning leadtimes are the userdefined parameters which are fixed, regardless of batch size. Manufacturing leadtimes in practice vary according to the status of workload and capacity 
available in the shop-floor. This causes the gap between the planned schedule and the real results in the shop-floor.

However, the actual leadtime for every item can not be found until detailed scheduling is performed. This results in the recurrent processes with large feedback loops as shown in Figure 1.

To resolve this problem, the manufacturing leadtime for each part is estimated as follows. First, the total processing time $T_{i j}$ per batch of part $P_{i}$ in a work-center $W_{j}$ is calculated as

$$
T_{i j}=n_{i} \cdot t p_{i j} \quad[\min .]
$$

where, $n_{i}$ is the batch quantity of part $P_{i}$ and $t p_{i j}$ is the processing time per unit of the part which is performed through several machines in the work-center. Then, the manufacturing leadtime of the part, $L T_{i}$, may be given by Eq. (2),

$$
L T_{i}=T_{B} \cdot N_{B}\left[\max _{\mathrm{j}} T_{i j}\right] \quad[\min .]
$$

where $T_{B}$ is the span of time bucket, $\max T_{i j}$ is the maximum total processing time over the work-centers through which the part $P_{i}$ is performed, and $N_{B}[X]$ means the minimum number of time buckets which includes the time length $X$.

Executing the time-phased parts explosion in MRP using the manufacturing leadtime obtained above, the production period to produce each part or product can be determined. Using the result, the load rate ${ }^{r_{j j k}}$ of each part $P_{i}$ in each workcenter $W_{j}$ for each production period (bucket) $B_{k}$ can be calculated by

$r_{i j k}=P T_{i j k} / T R_{j k}$

where $P T_{i j k}$ is the total processing time per batch of the part $P_{i}$ loaded in workcenter $W_{j}$ during bucket $B_{k}$, and $T R_{j k}$ is the available running time of work-center $W_{j}$ during $B_{k}$. Thus, the load rate of work-center $W_{j}$ in bucket $B_{k}$ is given by the sum of the load rates $r_{i j k}$ over all the parts loaded:

$$
r_{j k}=\sum_{i} r_{i j k}
$$

\subsection{Smoothing of overloads}

If any work-center is in the overload situation in any time period, some workloads should be moved into an alternative time period where capacity is available. To establish the procedure for smoothing of overloads, the loading states of the work- 
centers are classified into the following three groups, according to the load rate $r_{j k}$ given by Eq. (4).

Group 1: ${ }^{r_{j k}}>\alpha_{1}$ : $\quad$ overload state

Group 2: $\alpha_{1} \geqq r_{j k} \geqq \alpha_{2}: \quad$ adequate load state

Group 3: $r_{j k}<\alpha_{2}$ : extreme underload state

where $\alpha_{1}$ and $\alpha_{2}$ are upper and lower thresholds respectively, to distinguish the load states. Here, it should be noted that the load rates $r_{j k}$ are not necessarily exact, because they are not derived from detailed scheduling. Therefore, the value of $\alpha_{1}$ may not be $100 \%$, but some smaller value, for example, $65 \%$. In order to eliminate the feedback loop in Figure 1, the $\alpha_{1}$ should be determined as exactly as possible. These values are possibly varying depending on the manufacturing environment, and can be refined by experience or by feedback information from the scheduling module.

The change of time period to produce some part results in extension of leadtime for the part, and consequently, leadtime for the end product including the part as the component. It is therefore needed to know earliness of the end product, in order to see possibility of the change of production period for the part. Here, earliness of the product is given as follows,

$E_{i}=D_{i}-C M L T_{i}$

where $D_{i}$ is the time period from the present date to the due date of the end product $P_{i}$, and the $C M L T_{i}$ is the cumulative manufacturing leadtime of the end product $P_{i}$ which is defined as the longest leadtime from top to bottom of the product structure tree.

The parts allotted to be produced in each time period are classified into following three groups, according to earliness of the products including the parts as their components.

Group A : $E_{i}=0$ :

zero earliness

Group B : $E_{i} \leqq \boldsymbol{\beta} \cdot D_{i}: \quad$ adequate earliness

Group C: $E_{i}>\beta \cdot D_{i}$ :

too much earliness

where $\beta$ is the user-defined parameter like $\alpha_{1}$ and $\alpha 2$.

As a result, the parts to be loaded in each work-center in each time period are classified into 9 groups shown in Figure 2, based on the combination of three groups regarding the loading states of the work-center in Eq. (5) and three groups regarding earliness of the product in Eq. (7). 


\begin{tabular}{|c|c|c|c|}
\hline Earliness & $\begin{array}{c}\text { Group A } \\
\text { Zero }\end{array}$ & $\begin{array}{c}\text { Group B } \\
\text { Ade quate }\end{array}$ & $\begin{array}{c}\text { Group C } \\
\text { Too much }\end{array}$ \\
\hline $\begin{array}{c}\text { Group 1 } \\
\text { Overload }\end{array}$ & A-1 & B-1 & C-1 \\
\hline $\begin{array}{c}\text { Group 2 } \\
\text { Adequate }\end{array}$ & A-2 & B-2 & C-2 \\
\hline $\begin{array}{c}\text { Group 3 } \\
\text { Underload }\end{array}$ & A-3 & B-3 & C-3 \\
\hline
\end{tabular}

Figure 2 Part group based on loading states and earlinesses.

The smoothing procedure of overloads in the production planning level is proposed based on these groups of parts as follows.

Step 1 : If any work-center is in the overload situation (Group 1) at any time period, it is first tried to move some parts in the $\mathrm{C}-1$ situation into the immediately preceding period. In this case, the leadtime for the parts transferred will be extended, and therefore earliness of the relevant end product will be affected as well as the workload of the work-center with additional load at the preceding period. Thus, the change in the situation at the preceding period is examined, which may be caused by moving the loads. Then the transfers of the parts into the preceding period are tried in order of the situation C-3, C-2, B-3 and B-2 at the preceding period, until the overload situation at the current period will be resolved. In the case that the part to be moved has its child parts in the product structure, the child parts will be transferred backward by the time-phased parts explosion. As a result, if any overload situation is not caused in any time period, these transfers are adopted.

Step 2: If the overload situation at the time period under considerations can not be resolved by the procedure in Step 1, next consider the transfer of the parts in the B-1 situation into the preceding period. This transfer is tried in order of the situation B-3, B-2, A-3, and A-2 to be caused at the preceding period. These transfers can be adopted if any overload situation will not be caused at any time period by the subsequent transfers of the child parts.

Step 3: If any overload situation is caused at any time period by the transfers of the child parts in Step 1 or Step 2, the procedure of Step 1 or Step 2 is executed at the time period in which the overload has occurred.

Step 4: When the overload situation can not still be resolved through above steps, consider the case that the overload situation C-1 or B-1 will be caused at the preceding period by the transfer of the part in overload situation C-1 or B-1 at the current period into the preceding period. In these cases, the procedure from Step 1 to Step 3 will be executed for the preceding period.

Step 5: In the case that all of the above Steps end in failure, the overload situation is necessarily caused at some period. In this case, the transfer is adopted which 
causes the minimum overload, and the capacity is then adjusted to fit the load at the period where the overload exists.

\section{3}

\section{LOADING MODULE}

In the production planning level, the workload was allotted to each time bucket. The parts allotted to each bucket may practically be worked through more than one work-station according to their process routings.

It is therefore necessary to decide in which time period within the bucket and in which work-center each part should be loaded, so as to balance workload and capacity in any time period within the bucket and in any work-center.

For that purpose, the time bucket in the production planning level is subdivided into sub-buckets, the number of which is the maximum number of work-centers in which the parts are loaded within the time bucket, as shown in Figure 3. The subbucket is used as the unit of time in the loading in each work-center. The subbucket is also used to make the detailed production schedule for the parts loaded in each work-center within the sub-bucket. Thus the loading module can be integrated with the scheduling module.

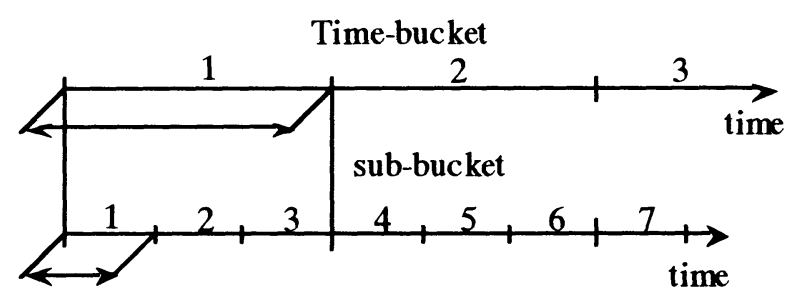

Figure 3 Time management in production planning and loading stages.

In order to make the feasible loading plan into each work-center in each subbucket, the load rate of the work-center in the sub-bucket is calculated. However the actual load rate can not be determined until the detailed production schedule in the work-center within the sub-bucket is given. Here, the load rate is therefore tentatively estimated regardless of the waiting time. Thus the load rate per batch of the part is given as follows.

$$
L R_{i j k}=\frac{L_{i j k} \cdot n_{i}}{C_{j k}}
$$

where $L R_{i j k}$ is the load rate per batch of part $P_{i}$ in the work-center $W_{j}$ within the sub-bucket $S B_{k}, L_{i j k}$ is the total sum of the standard processing times on machines included in the work-center $W_{j}$ per unit of part $P_{i}$ loaded in the sub-bucket $S B_{k}, n_{i}$ 
is the batch quantity of part $P_{i}$, and $C_{j k}$ is the sum of the available running times of all the machines included in the work-center ${ }^{W_{j}}$ within the sub-bucket $S B k$.

Loading and smoothing of the workload into work-centers are performed according to following steps.

Step 1 ( loading ) : The parts allotted into a time bucket in the production planning module are loaded forward from the first sub-bucket within the time bucket into the required work-centers according to the production routing of each part.

Step 2 ( smoothing of overloads ): If any work-center is in the overload situation at any sub-bucket, the part which is manufactured through the work-centers less than the number of sub-buckets within the time bucket is selected as a candidate to be moved to later sub-bucket. In the case there are more than one part in such situation, the part to be moved is selected in increasing order of the number of work-centers to be successively loaded. When the overload situation can be resolved in all sub-buckets, it is possible to proceed to the next step. On the other hand, if the resolution of overload situation is not possible in this way, information on the load rate in the overload situation will be fed back to the production planning module in order to change the time bucket for the overload work. In this step, the overload situation can be recognized by the following equation using the load rate given by Eq. (8).

$$
L R_{j k}=\sum_{i} L R_{i j k}>\gamma_{1}
$$

where $\Sigma$ is the sum of load rates of parts to be loaded into the work-center $W_{j}$ in sub-bucket $S B_{k}$ and $\gamma_{1}$ is the user-defined parameter to distinguish the load states. Here, $\gamma_{1}$ may not be $100 \%$, but some smaller value, because the load rates $L R_{i j k}$ were estimated without regard to queue time. The value of $\gamma_{1}$ can be refined by experience or feedback information out of detailed scheduling.

Step 3 (smoothing of work loads) : If imbalance exists between work-centers in each sub-bucket, the smoothing procedure is carried out in such a way as Step 2. If these steps result in the extreme underload situation given by the load rate $L R_{j k}$ $<\gamma 2$ in every sub-bucket, the span of sub-buckets should be shortened so as to be appropriate-load situation, $\gamma_{1}>L R_{j k}>\gamma_{2}$. As a result, the span of time bucket in the production planning level can be shortened as well, which makes it possible to shorten the production leadtime of products.

\section{SCHEDULING MODULE}

In order to perform the production of the parts loaded to the work-center within the sub-bucket along the loading schedule and to realize leadtimes as shorter as possible, the appropriate schedule should be made so as to minimize the queue time, whose method may be found in many studies like Oba et al.(1993). 
If it is found that the production of parts loaded can not be completed within the sub-bucket in this stage, the information of load rates is fed back to the loading module and rearrangement of the loads is carried out using the actual load rates based on detailed scheduling. The feedback information of actual load rates may be utilized to refine the threshold value of $\gamma_{1}$ in the loading level as well as $\alpha_{1}$ in the production planning level.

\section{NUMERICAL EXAMPLE}

The six kinds of end products $\mathrm{X}, \mathrm{Y}, \mathrm{Z}, \mathrm{H}, \mathrm{J}, \mathrm{T}$ are produced in the example. The detailed bills of materials are not described here owing to limited space, but each product structure comprises some of six kinds of subassemblies(A,B,C,D,L,K,S) and twelve kinds of parts(d,p,f,v,u,e,m,g,n,o,r,q) as shown in Figure 4 as a couple of examples. In Figure 4, an alphabet shows a part, a subassembly or a product and a numeral attached to the alphabet is the number of the part required to produce a unit of its parent. In the production system, there are three work-centers WC\#1, WC\#2 and WC\#3 for assembly, and three work-centers WC\#4, WC\#5 and WC\#6 for machining, each of which comprises four machines. Each part is finished through two or three work-centers in which the processes between three and six are performed. The standard processing time of each process is distributed between 2 and 28 minutes.

Figure 5 shows the result of the time-phased parts explosion in MRP based on the master production schedules(MPS) for the products X,Y,Z,H,J,T and their bills of materials. In this figure, an alphabet shows a part, a subassembly or a product and a numeral attached to the alphabet is the required volume. The span of time bucket is a week( 2880 minutes $)$.
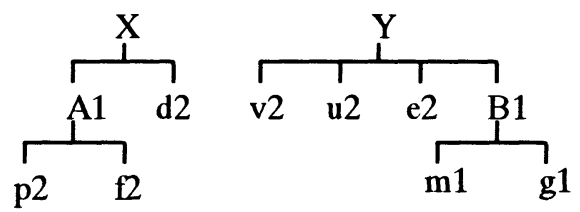

Figure 4 Example of product structure trees of products $\mathrm{X}$ and $\mathrm{Y}$. 


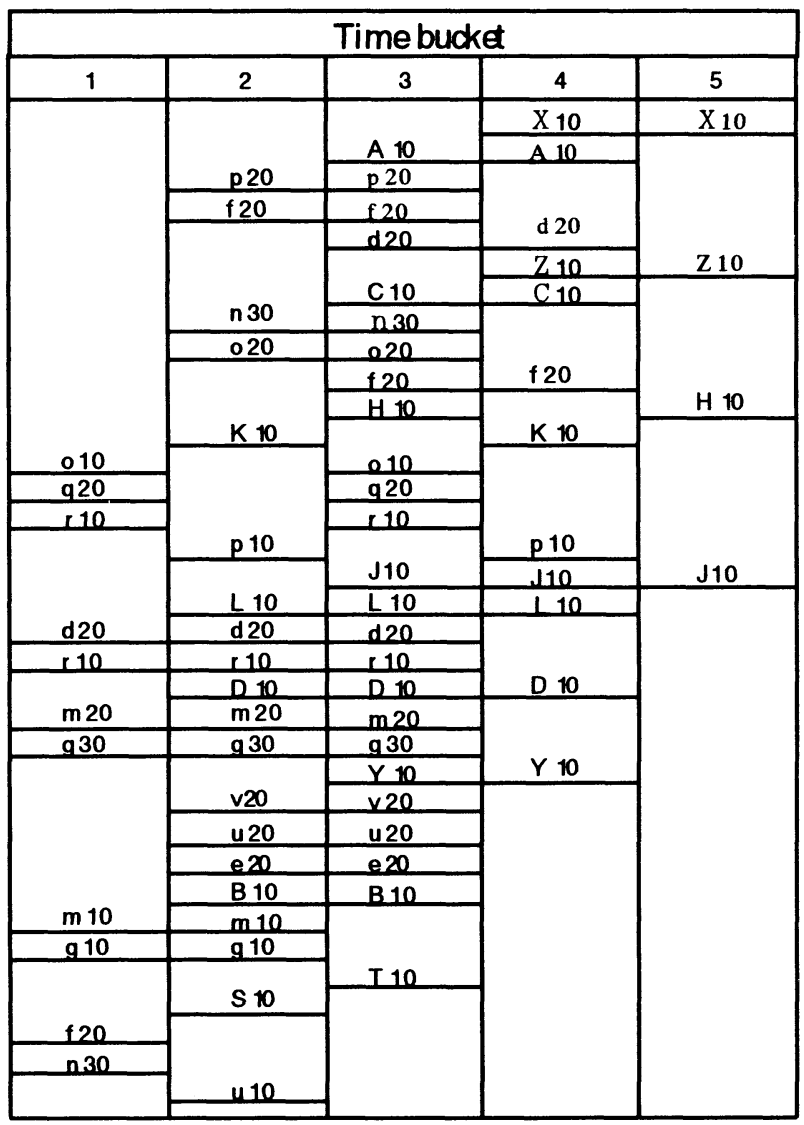

Figure 5 Time-phased parts explosion in MRP.

This result may be accepted because there is no overload situation in any workcenter in any time bucket. We can then directly proceed to the stage of loading. Figure 6(a) shows the result of loading in which the workload is allotted to each work-center in each sub-bucket of time. This figure gives the load rates in percentage. In this figure, some overload situations are found in the hatched subbuckets in each work-center. Here, the threshold to identify the overload status $\alpha_{1}$ is put at 65 percent. These overload situation can be resolved according to the smoothing procedure in the loading module and the result is shown in Figure 6(b) in which the shadowed numerals show the load rates changed with the workloads transfer from or to another sub-bucket. 


\begin{tabular}{|c|c|c|c|c|c|c|c|c|c|c|c|c|c|c|c|}
\hline & bucket & \multicolumn{3}{|c|}{1} & \multicolumn{3}{|c|}{2} & \multicolumn{3}{|c|}{3} & \multicolumn{3}{|c|}{4} & \multicolumn{2}{|c|}{5} \\
\hline & sub-budket & 1 & 2 & 3 & 4 & 5 & 6 & 7 & 8 & 9 & 10 & 11 & 12 & 13 & 14 \\
\hline \multirow{6}{*}{ 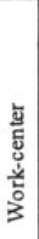 } & $\# 1$ & & & & 13 & 18 & 13 & 29 & 22 & 26 & 31 & 45 & 26 & 19 & 9 \\
\hline & $\# 2$ & & & & 15 & 65 & 6 & 25 & 79 & 6 & 41 & 62 & 0 & 6 & 22 \\
\hline & $\# 3$ & & & & 54 & 9 & 13 & 66 & 38 & 9 & 52 & 38 & 22 & 10 & 9 \\
\hline & $\# 4$ & 28 & 29 & 40 & 52 & 40 & 50 & 47 & 58 & 42 & 17 & 9 & 0 & & \\
\hline & $\# 5$ & 26 & 72 & 0 & 45 & 58 & 8 & 59 & 74 & 5 & 10 & 14 & 0 & & \\
\hline & $\# 6$ & 50 & 8 & 16 & 66 & 48 & 12 & 71 & 42 & 25 & 0 & 5 & 8 & & \\
\hline
\end{tabular}

(a) before smoothing

\begin{tabular}{|c|c|c|c|c|c|c|c|c|c|c|c|c|c|c|c|}
\hline & \multirow{2}{*}{\begin{tabular}{|c|} 
bucket \\
sub-bucket
\end{tabular}} & \multicolumn{3}{|c|}{1} & \multicolumn{3}{|c|}{2} & \multicolumn{3}{|c|}{3} & \multicolumn{3}{|c|}{4} & \multicolumn{2}{|c|}{5} \\
\hline & & 1 & 2 & 3 & 4 & 5 & 6 & 7 & 8 & 9 & 10 & 11 & 12 & 13 & 14 \\
\hline \multirow{6}{*}{ 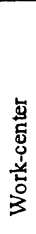 } & $\# 1$ & & & & 13 & 18 & 13 & 29 & 22 & 26 & 31 & 45 & 26 & 19 & 9 \\
\hline & $\# 2$ & & & & 15 & 43 & 28 & 25 & 56 & 29 & 41 & 42 & 20 & 6 & 22 \\
\hline & \# 3 & & & & 37 & 26 & 13 & 48 & 39 & 26 & 52 & 38 & 22 & 10 & 9 \\
\hline & $\# 4$ & 28 & 29 & 40 & 52 & 30 & 60 & 47 & 41 & 60 & 17 & 9 & 0 & & \\
\hline & $\# 5$ & 26 & 36 & 36 & 45 & 44 & 22 & 49 & 56 & 33 & 10 & 14 & 0 & & \\
\hline & \# 6 & 36 & 22 & 16 & 54 & 51 & 21 & 52 & 51 & 35 & 0 & 5 & 8 & & \\
\hline
\end{tabular}

(b) after smoothing

Figure 6 Load rate in each work-center.

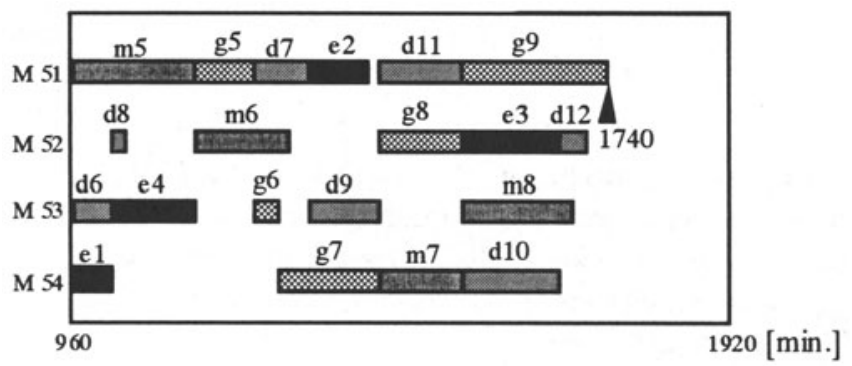

Figure 7 Schedule in WC\#5 in sub-bucket SB8 after smoothing.

Production scheduling in each work-center in each sub-bucket is carried out based on the result of loading and smoothing in the loading module. As an example, the detailed schedule in the work-center WC\#5 in the sub-bucket SB8 after smoothing is shown in Figure 7. In this figure, the alphabets are parts which are loaded to be machined in the work-center WC\#5 in the sub-bucket SB8, and the numerals attached to each part are the operation numbers according to whose precedence relations the part is machined. The precedence relations of operations for each part are partially determined by process planning and the operations are not necessarily processed in increasing order. 


\section{CONCLUSION}

In this study, a new procedure for decision-making has been proposed to integrate production planning with loading and scheduling. Difficulties of this problem are essentially caused by the strongly connected relationship between production planning, loading and scheduling like that the results of decision made in the lower level of scheduling or loading are needed for the decision in higher level of production planning. The strongest point of this study is that the architecture of decision-making has been given to solve this difficult problem.

\section{REFERENCES}

Matoba, H., Ohnari, H. and Enomoto, M. (1993), Production Planning System using Dynamic MRP. in 'Proceeding of Production Scheduling Symposium', Tokyo, pp. 99-102. JSME (in Japanese).

Oba, F., Jigami, O. and Fushimi, J. (1993), Loading and Scheduling in Consideration of Alternative Process Sequences. in 'Proceedings of Production Scheduling Symposium', Tokyo, pp. 141-145. JSME (in Japanese).

Scott, B. (1994), Manufacturing Planning Systems, chapter 22. McGraw-Hill, UK.

\section{BIOGRAPHY}

Fuminori Oba is a professor at Hiroshima University since 1988 . He received his master degree from University of Osaka Prefecture in 1968, and his doctor degree from Osaka University in 1975. His current interests include design of manufacturing systems, scheduling, production management.

Takeshi Murayama is an associate professor at Hiroshima University since 1994. He received his master degree from University of Osaka Prefecture in 1986, and his doctor degree from Hiroshima University in 1993. His current interests include $\mathrm{CAD} / \mathrm{CAM}$, concurrent engineering, assembly planning, and environmentally conscious design and manufacturing.

Dianying Ma is an engineer at IHI Packaged Boiler Fabricators Co., Ltd. since 1997. She received her master degree from Beijing University of Aeronautics and Astronautics in 1982. Her current interests include scheduling, process planning, autonomous manufacturing systems, and petri nets. 